\title{
TSUNAMI INVERSION OF FAULT DISPLACEMENTS BASED ON SPARSE MODELING
}

\author{
Tetsushi KURITA ${ }^{1}$ \\ ${ }^{1}$ Member of JAEE, Tokyo Electric Power Services Co., Ltd., Tokyo Japan, kurita@tepsco.co.jp
}

\begin{abstract}
A study on tsunami inversion analysis was conducted using Lasso regression, which is a typical approach to sparse modeling. Characteristics of spatiotemporal distribution of the fault slips were then analyzed. Sparse modeling is characterized by yielding obtained solutions that contribute significantly to the output of the model. This feature is useful for investigating the development of prediction models. This paper applies a tsunami inversion analysis based on sparse modeling to the 2011 Tohoku Earthquake Tsunami and analyzes the characteristics of the fault slips.
\end{abstract}

Key Words: sparse modeling, Lasso, tsunami, inversion, fault slip

\section{INTRODUCTION}

To understand the characteristics of a tsunami caused by an earthquake, it is important to understand what kind of fault movement occurred at the hypocenter. Tsunami inversion refers to the process of estimating the slip displacement of earthquake source faults from the traces of the tsunami left in the coastal areas, as well as the tidal records. Analyzing the fault slip model estimated using tsunami inversion and understanding its features can produce important information for future prediction problems necessary for tsunami resistant design. In the 2011 Tohoku Earthquake Tsunami, since a large number of observation records were obtained, highly accurate source fault models can be obtained by tsunami inversion ${ }^{11}$.

On the other hand, a method called sparse modeling is used in the field of machine learning to construct mathematical models from various big data such as medical service and life logs ${ }^{2}$. It is also used in the field of image processing for compression and decompression ${ }^{3)}$. Sparse means not dense, and sparse modeling (or sparse estimation) is a modeling method that uses the property assumes that most regression coefficients are zero. The advantage of sparse modeling is that model selection can be performed simultaneously with regression, since the regression coefficients of low-contributing parameters for the output of the target mathematical model are automatically zero. These advantages are also useful properties in tsunami inversion. Thus, applying sparse modeling to tsunami inversion is considered significant. It is important to model the construction of physical models that explain natural phenomena using as few parameters as possible. The idea here is that the resulting model will be better able to be used for predictive purposes ${ }^{4)}$.

It is thought that applying the method of sparse modeling to the problem of tsunami inversion will make some level of predictive analysis possible. Therefore, in this study, tsunami inversion was conducted using Lasso regression, which is a typical form of sparse modeling, and analyzed the characteristics of the spatiotemporal distribution of slips in earthquake source faults are analyzed. The 
purpose of this study is to propose a method for efficiently understanding the fault motion that affects the tsunami. To solve such problems, if we use sensitivity analysis to examine the impact on the tsunami exerted by each spatiotemporal slip constituting the fault motion, it becomes possible to search for a combination of spatiotemporal slips that have a large impact on the tsunami. However, if the fault model is complicated and the degree of freedom is large, much work and time will be required. Sparse modeling, by contrast, is advantageous insofar as it allows for the automatic extraction of those parameters that have a large impact on the tsunami.

\section{APPLICATION OF SPARSE MODELING TO TSUNAMI INVERSION}

This paper presents two approaches to tsunami inversion: the conventional approach and sparse modeling approach, and uses a comparison of these two to demonstrate the applicability and usefulness of sparse modeling approach.

\subsection{Conventional Tsunami Inversion}

This study examines the problem of finding the slip displacements of the fault using the same formulation as Kurita ${ }^{5)}$. It is assumed that the fault plane is comprised of multiple subfaults separated in a grid. Here, tsunami inversion is formulated in vector notation for unknown quantities and observable quantities, and is defined as follows: $\|\cdot\|_{1}$ means L1 norm $\left(\|\mathbf{z}\|_{1}=\sum_{k}\left|z_{k}\right|\right),\|\cdot\|_{2}$ means L2 $\operatorname{norm}\left(\|\mathbf{z}\|_{2}=\sqrt{\sum_{k} z_{k}^{2}}\right)$.

In the conventional inversion problem, the solution is obtained by the nonnegative least squares method as follows. The reason for adding the nonnegative condition to the least squares method is that without it, the formula could result in a negative slip result. A negative slip would indicate that movement occurred in the opposite direction to the slip direction, and would be unnatural. Hence, the nonnegative condition is added.

$$
\min \|\mathbf{G} \cdot \mathbf{X}-\mathbf{Y}\|_{2}^{2} \quad \text { subject to } \mathbf{X} \geq \mathbf{0}
$$

where G : Green's function matrix, $\mathbf{X}$ : fault slip vector, $\mathbf{Y}$ : observation vector. The observed quantities are crustal deformation (permanent displacement) and tsunami water level time history.

The multi-time window model ${ }^{8)}$ was used as the slip model of the earthquake source fault, as was done in previous studies ${ }^{5}$. This method adds the Green's functions, taking into consideration the rupture time and the time delay of the time window, when performing the linear tsunami analysis. It was assumed that the rupture propagation proceeded concentrically from the hypocenter position as determined by the Japan Meteorological Agency. In this study, the constraint condition of smoothing is not provided to suppress the difference between adjacent spatiotemporal slips.

\subsection{Formulation of Sparse Modeling}

To perform sparse modeling, a Lasso (Least absolute shrinkage and selection operator) type $\mathrm{e}^{7)}$ evaluation function in which the unknown parameters are L1 regularized is applied as follows:

$$
\min \|\mathbf{G} \cdot \mathbf{X}-\mathbf{Y}\|_{2}^{2}+\lambda\|\mathbf{X}\|_{1}
$$

where $\lambda$ is the regularization parameter $(\lambda \geq 0)$. The L1 regularization case is called Lasso regression, as shown in equation (2), and the L2 regularization case formulated with the square of unknown parameters is called Ridge regression. Regularization is a method used to prevent overfitting 
to observed data.

In the case of L2 regularization such as Ridge regression, the estimated value can be obtained by partially differentiating the evaluation function to be minimized with unknown parameters, but in the case of L1 regularization, partial differentiation by unknown parameters is not possible. Therefore, the solution can be obtained using algorithms such as CD (Coordinate Descent) ${ }^{8), 9)}$ or ADMM (Alternate Direction Method of Multipliers) ${ }^{10}$. In both methods, the optimal solution is obtained by iterative calculation while controlling the value of the unknown parameter with the soft-thresholding function as shown in Eq. (3). The image of the soft-thresholding function is shown in Fig. 1.

$$
S(x, \lambda)=\left\{\begin{array}{cc}
x-\lambda & (x>\lambda) \\
0 & (|x| \leq \lambda) \\
x+\lambda & (x<-\lambda)
\end{array}\right.
$$

In the algorithm for Lasso regression, the solution is obtained by sequentially correcting the estimated values of the unknown parameters. In the sequential calculation, a soft-thresholding function is applied to new candidate values in the step of updating the unknown parameters of the model to fit the observed data. At this time, the role of the soft-thresholding function is as follows: output is replaced by zero if the input value falls under the threshold, and if the input value exceeds the threshold, the original value reduced by the threshold amount is output. The above operation is repeated until the solution converges.

In sparse modeling in tsunami inversion obtained by the above formulation, the slip displacement of a subfault with a small contribution to the model output is determined to be zero. That is, the subfault where the slip displacement is zero has been deemed to be unnecessary in modeling.

As an example, Fig. 2 shows the Lasso type sparse solutions using the soft-thresholding function of Eq. (3). Here, the number of time windows is one. From the figure, negative values can be seen as the slip displacements. This means that slippage in the reverse direction to the slip direction has occurred, and it is not appropriate as a fault model. Thus, in this study, the soft-thresholding function like the following equation so that the negative slip does not occur is proposed. This function behaves as shown in $S^{\prime}(x, \lambda)$ in Fig. 1.

$$
S^{\prime}(x, \lambda)=\left\{\begin{array}{cc}
x-\lambda & (x>\lambda) \\
0 & (x \leq \lambda)
\end{array}\right.
$$

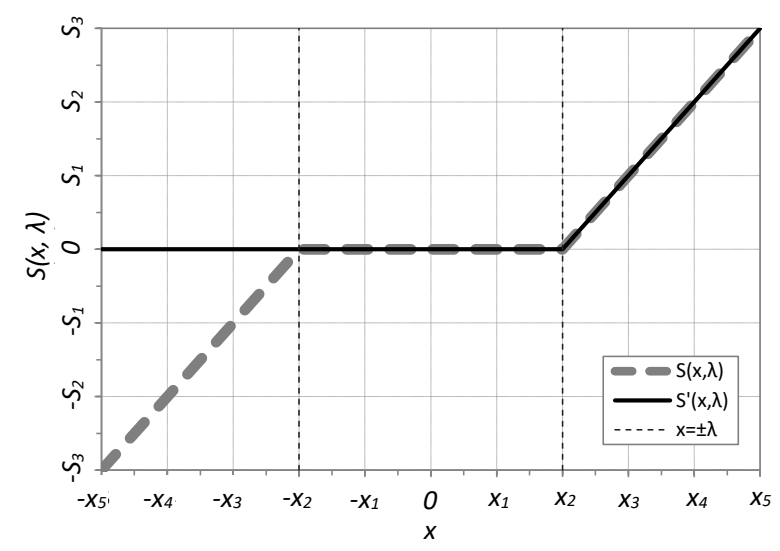

Fig. 1 Soft-thresholding function 


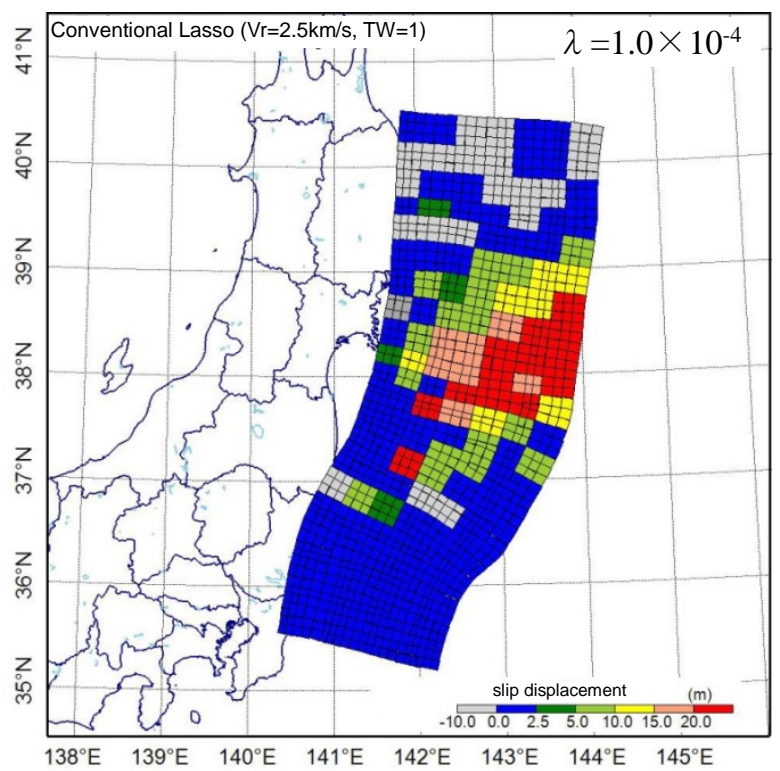

Fig. 2 Final fault slip distribution obtained using a conventional soft-thresholding operator

\section{TSUNAMI INVERSION RESULT USING LASSO REGRESSION}

\subsection{Sparse Solution of Fault Slip}

In this study, sparse modeling was applied to tsunami inversion analysis for the 2011 Tohoku Earthquake Tsunami. The analysis conditions for performing inversion were set as follows based on previous studies ${ }^{5}$. The rupture velocity was $2.5 \mathrm{~km} / \mathrm{s}$, the number of time windows was 5 , and the length of one time window was 30 seconds. The earthquake source fault is comprised of subfault blocks divided into 147. The number of unknowns is the number of subfault blocks (147 blocks) $\times$ number of time windows (5 windows) $=735$. The data of crustal deformation (permanent displacement) and tsunami water level time history published by various organizations ${ }^{11)-16)}$ were used as observations. As observations, 395 sites $\times 3$ components are used as crustal deformation (permanent displacement), and tsunami water level time history data is used in total of 14,522 steps at 40 sites. The duration of the tsunami water level time history to be used was from the beginning of the rupture to the first direct wave at each observation site.

Lasso regression was performed by changing the value of the regularization parameter $(\lambda)$ using the soft-thresholding function of Eq. (4). The final fault slip distribution estimated by Lasso under nonnegative conditions is shown in Fig. 3. In the case of $\lambda=1.0 \times 10^{-2}$, a result with no slip was obtained. Also, it agrees with the result obtained by the least squares method of ordinary nonnegative condition at $\lambda=1.0 \times 10^{-8}$ or less. It can be seen that the final slip displacements of the source fault increase as the regularization parameter decreases.

In the case of $\lambda=1.0 \times 10^{-3}$, where the value of the regularization parameter is the largest among the calculation cases in which the solution of slip distribution is obtained, the slips are distributed only in a relatively deep region slightly north of the rupture starting point. As the value of the regularization parameter decreases, the subfault blocks where the slip occur are expanding. In particular, slipping along the trench axis tends not to appear unless the value of the regularization parameter is small enough.

When the value of the regularization parameter is changed, the change process of the residual sum of squares between observed data and calculated values from Lasso estimation and the number of nonzero parameters is shown in Fig. 4. This shows that the residual sum of squares increases as the value of the regularization parameter increases. Also, the number of nonzero parameters decreases accordingly. 


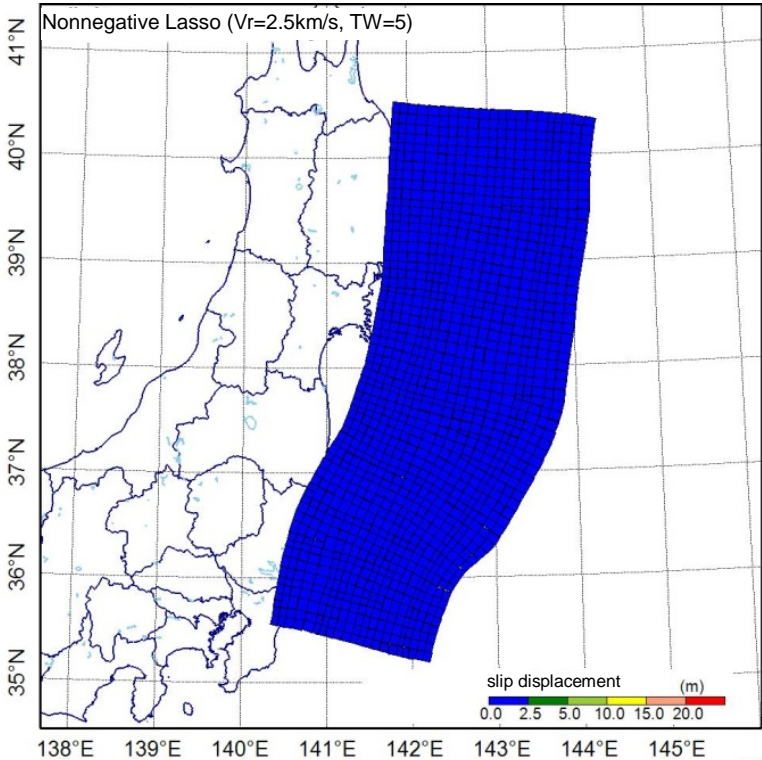

(1) $\lambda=1.0 \times 10^{-2}$

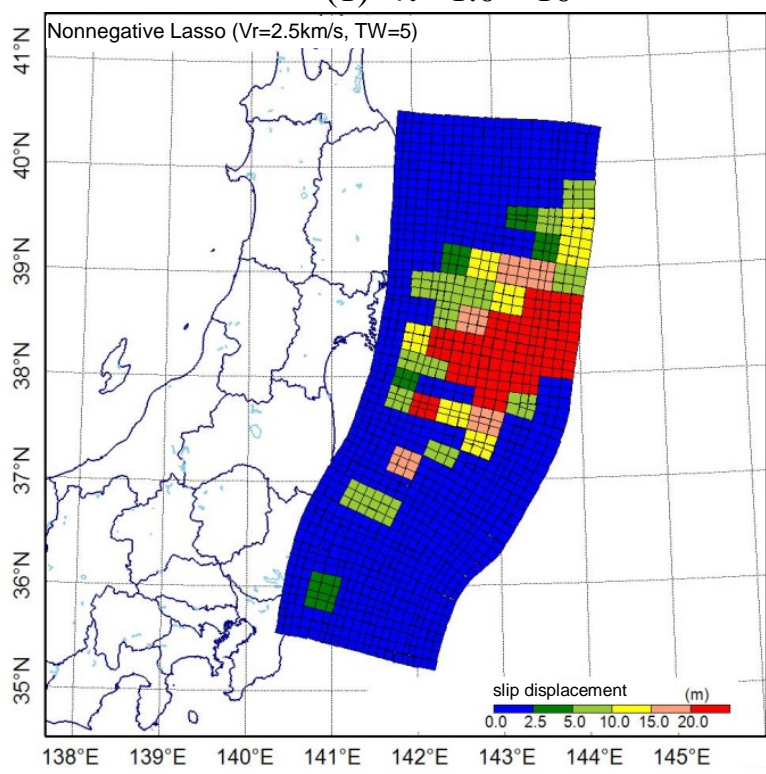

(3) $\lambda=1.0 \times 10^{-4}$

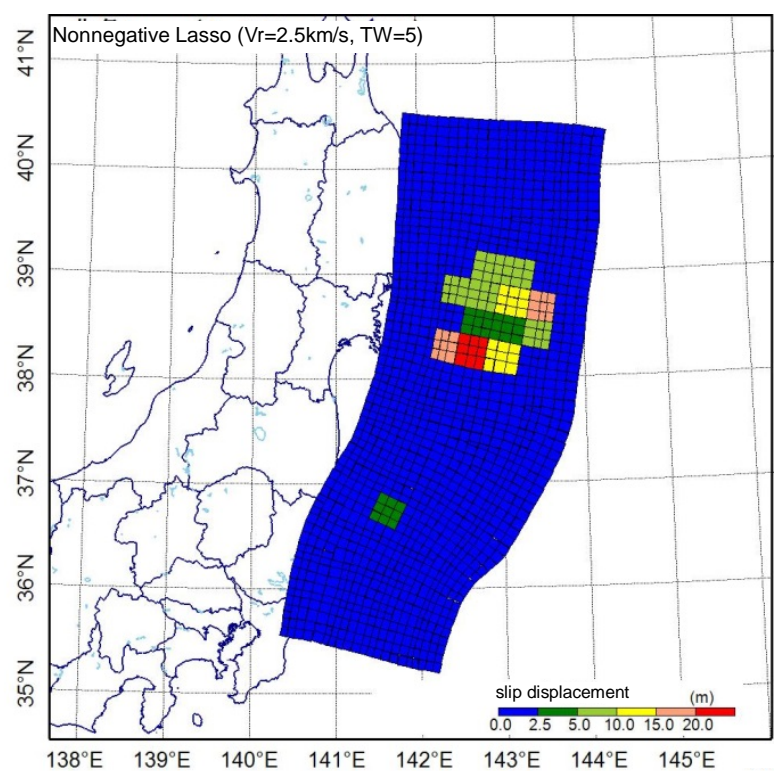

(2) $\lambda=1.0 \times 10^{-3}$

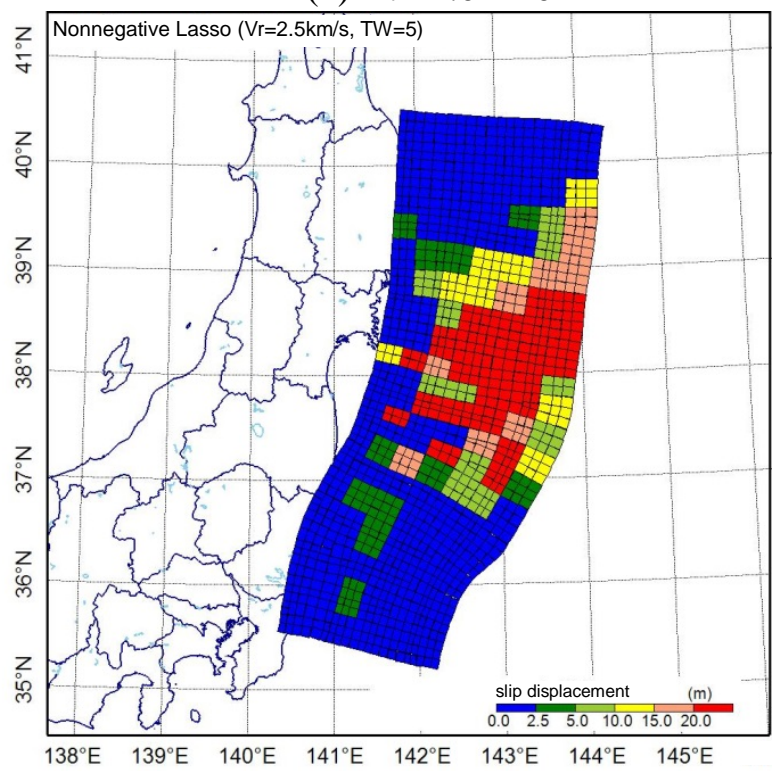

(4) $\lambda=1.0 \times 10^{-5}$

Fig. 3 Final fault slip distribution estimated by Lasso under nonnegative conditions (1)

Next, the estimation models were evaluated according to AIC $_{\lambda}^{\text {Lasso } 17)}$. Here, the parameters of the model are the active set of unknowns $\mathbf{X}$. Figure 5 shows the relationship between the values of the regularization parameters and $\mathrm{AIC}_{\lambda}^{\text {Lasso }}$. In addition, since two different physical quantities of crustal deformation (permanent displacement) and tsunami water level time history are used as observation quantities, $\mathrm{AIC}_{\lambda}^{\text {Lasso }}$ is calculated for each observation data. The figure shows that the estimation result when the regularization parameter is small ( $\lambda=1.0 \times 10^{-8}$ or less) is more appropriate in the evaluation result by $\mathrm{AIC}_{\lambda}^{\text {Lasso }}$. 

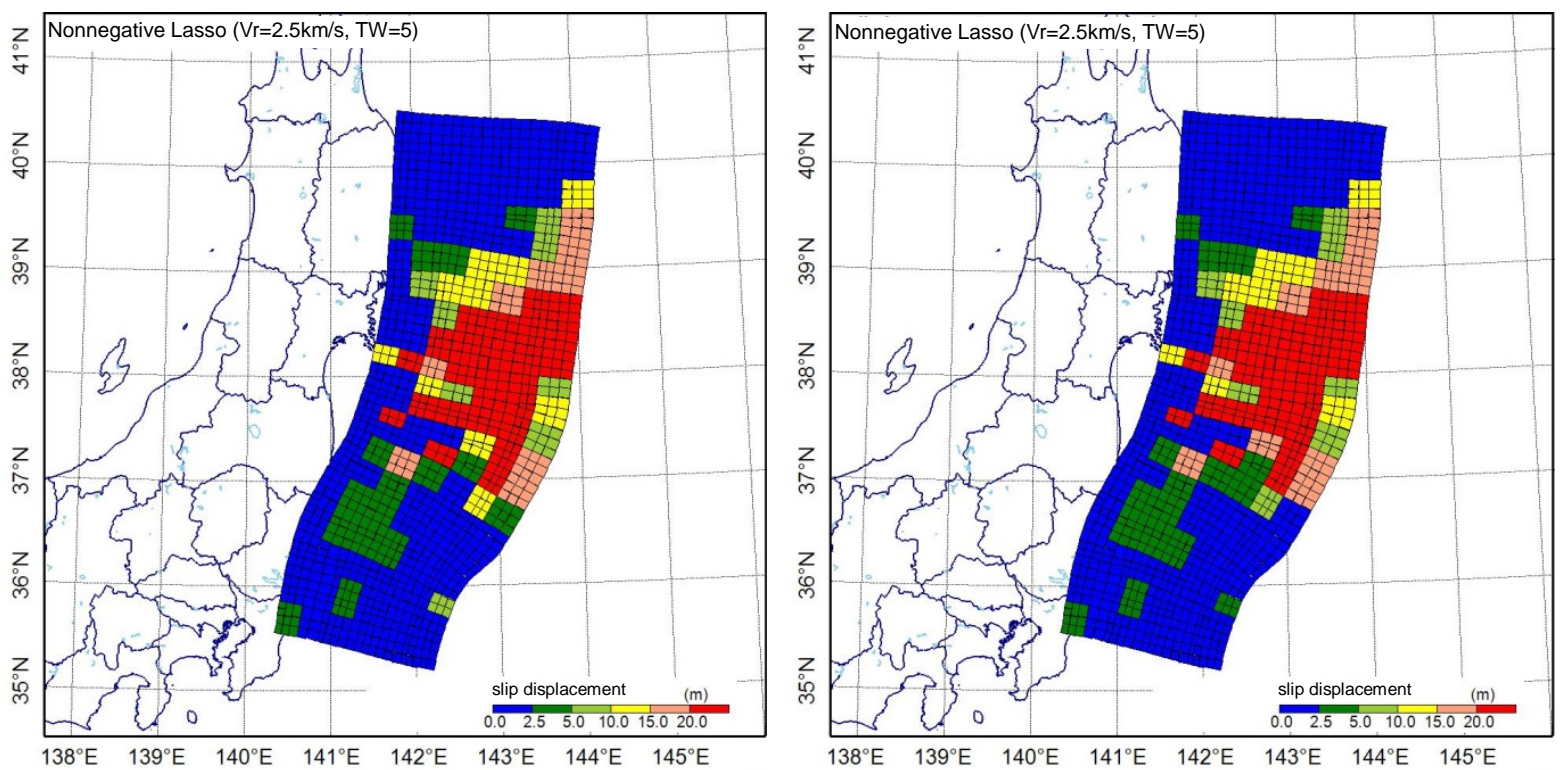

(5) $\lambda=1.0 \times 10^{-6}$

(6) $\lambda=1.0 \times 10^{-7}$

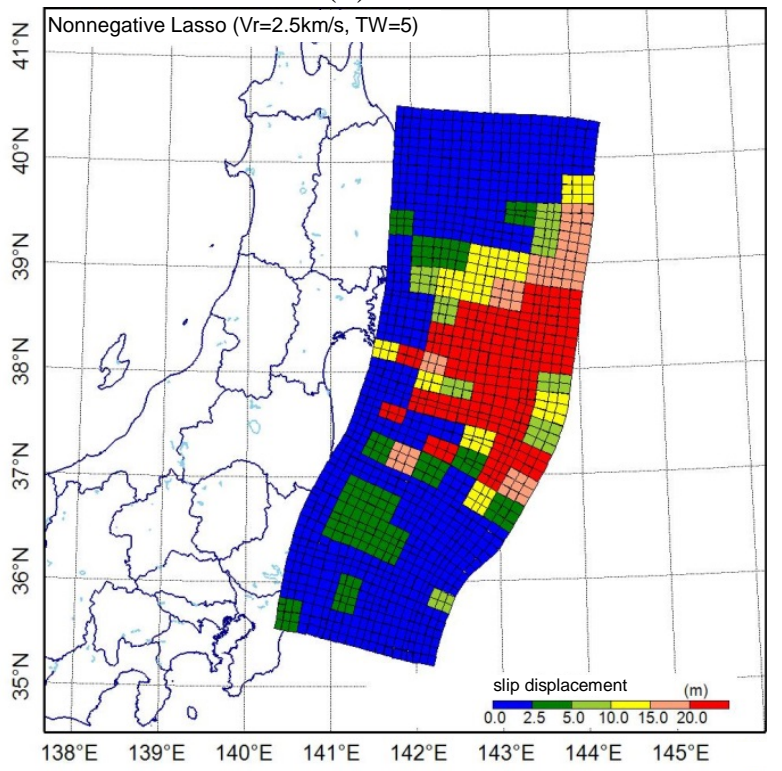

(7) $\lambda=1.0 \times 10^{-8}$

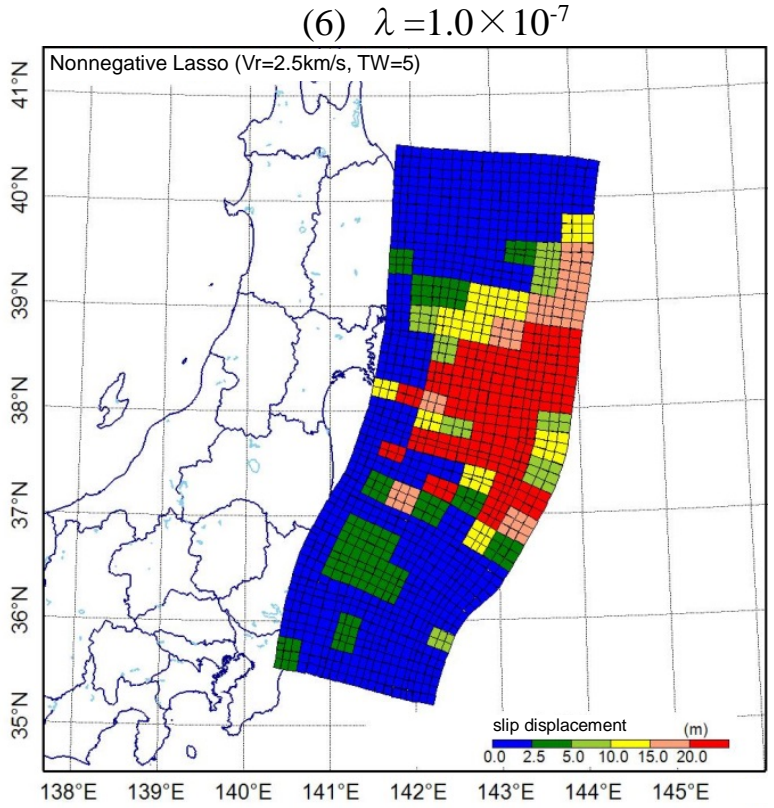

(8) $\lambda=1.0 \times 10^{-9}$

Fig. 3 Final fault slip distribution estimated by Lasso under nonnegative conditions (2)

\subsection{Solution Path of Fault Slip}

Although Fig. 3 shows the values of the regularization parameters and the final fault slip displacements, in this study, since the multi-time window is used as the source slip model, it is necessary to regard the Lasso estimated value as the spatiotemporal distributions. Figure 6 shows the Lasso regression estimate of the time change of the slip at each subfault block. If the value of the regularization parameter decreases by a certain degree or more, subfault blocks with large slippage are almost the same in the end, even if the regularization value changes. For example, in Fig. 6 (2) and (3), subfault blocks No. 12 to No. 14, No. 39 to 40, and No. 66 to 68 share the final slip displacements of more than $25 \mathrm{~m}$. However, when looking at all faults, the temporal variations of the slip displacements change depending on the value of the regularization parameter. 


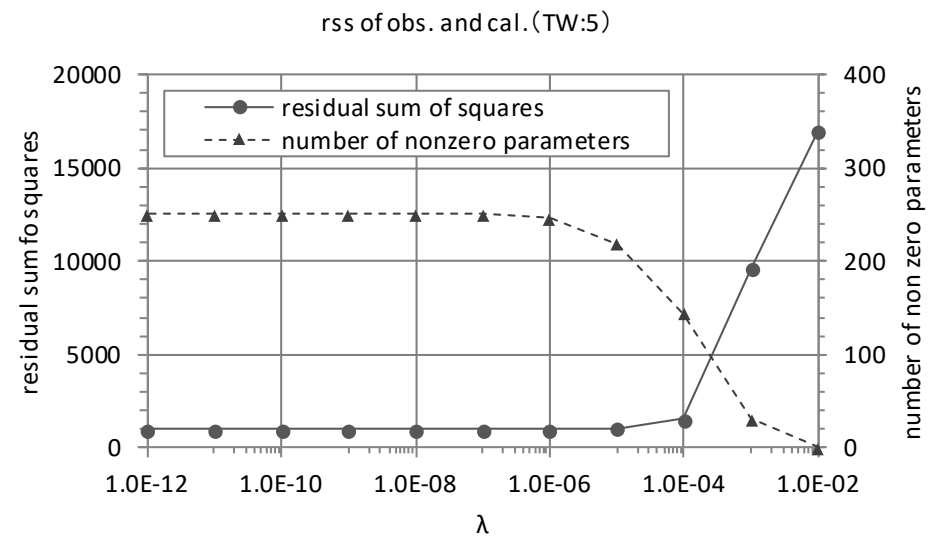

Fig. 4 Relationships between the variation of $\lambda$ and the residual sum of squares, also between the variation of $\lambda$ and the number of nonzero parameters

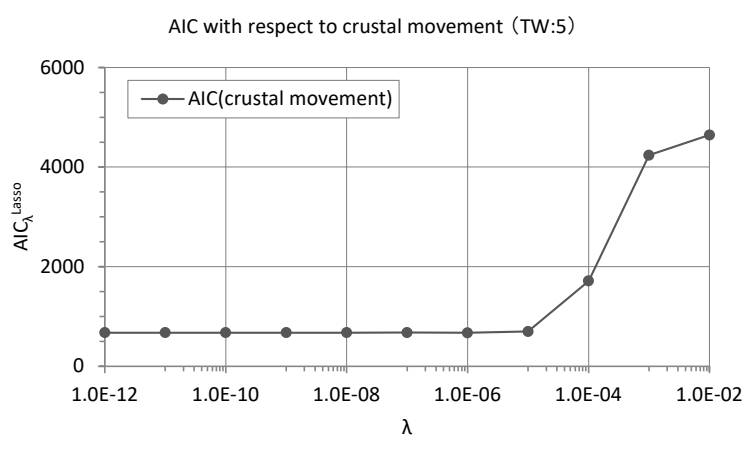

(1) $\mathrm{AIC}_{\lambda}^{\text {Lasso }}$ on crustal deformation (permanent displacement)

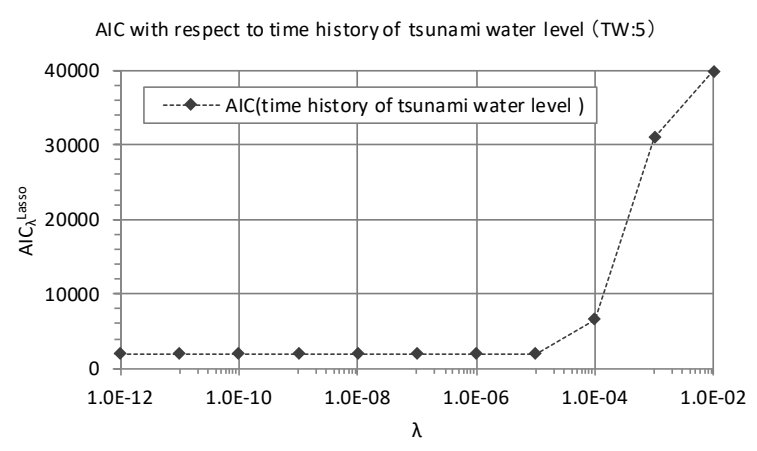

(2) $\operatorname{AIC}_{\lambda}^{\text {Lasso }}$ on tsunami water level time history

Fig. 5 Relation between changing $\lambda$ and $\operatorname{AIC}_{\lambda}^{\text {Lasso }}$

The solution path was obtained to investigate the state of the spatiotemporal change of the Lasso regression estimated value by the value of the regularization parameter. Figure 7 shows the solution path of the fault slip using Lasso regression for each time window. Here, we focus on 5 subfault blocks with a relatively large slip. The figure shows that the slip displacements change depending on the value of the regularization parameter even in the same fault block, in the same time window. It is also understood that the magnitude relation of the slip displacement with other blocks may be replaced with one another. In particular, block No. 092 of time window: 1 and block No. 121 of time window: 3 are drastically changed by the value of the regularization parameter. This phenomenon can also be confirmed by the time changing of slip displacements of the subfault block in Fig. 6. For block No. 092 (solid frame in the figure), the time functions of the slip are drastically different between $\lambda=1.0 \times$ $10^{-3}$ and $\lambda=1.0 \times 10^{-8}$. Similarly, for block No. 121 (broken line frame in the figure), the time functions of the slip at $\lambda=1.0 \times 10^{-4}$ and at $\lambda=1.0 \times 10^{-8}$ are vastly different. Slipping the fault in the temporally backward time window and slipping the spatially distant fault block has the same effect on the tsunami water level at the observation point. By setting the regularization parameters, places appear where the amount of slip fluctuates, as described above.

As mentioned above, although sparse modeling can obtain the subfault blocks with a large impact on the tsunami waveform, the degree of impact may change depending on the value of the regularization parameter, so it should be carefully monitored. It is therefore important to confirm the fluctuation of solution by solution path. 


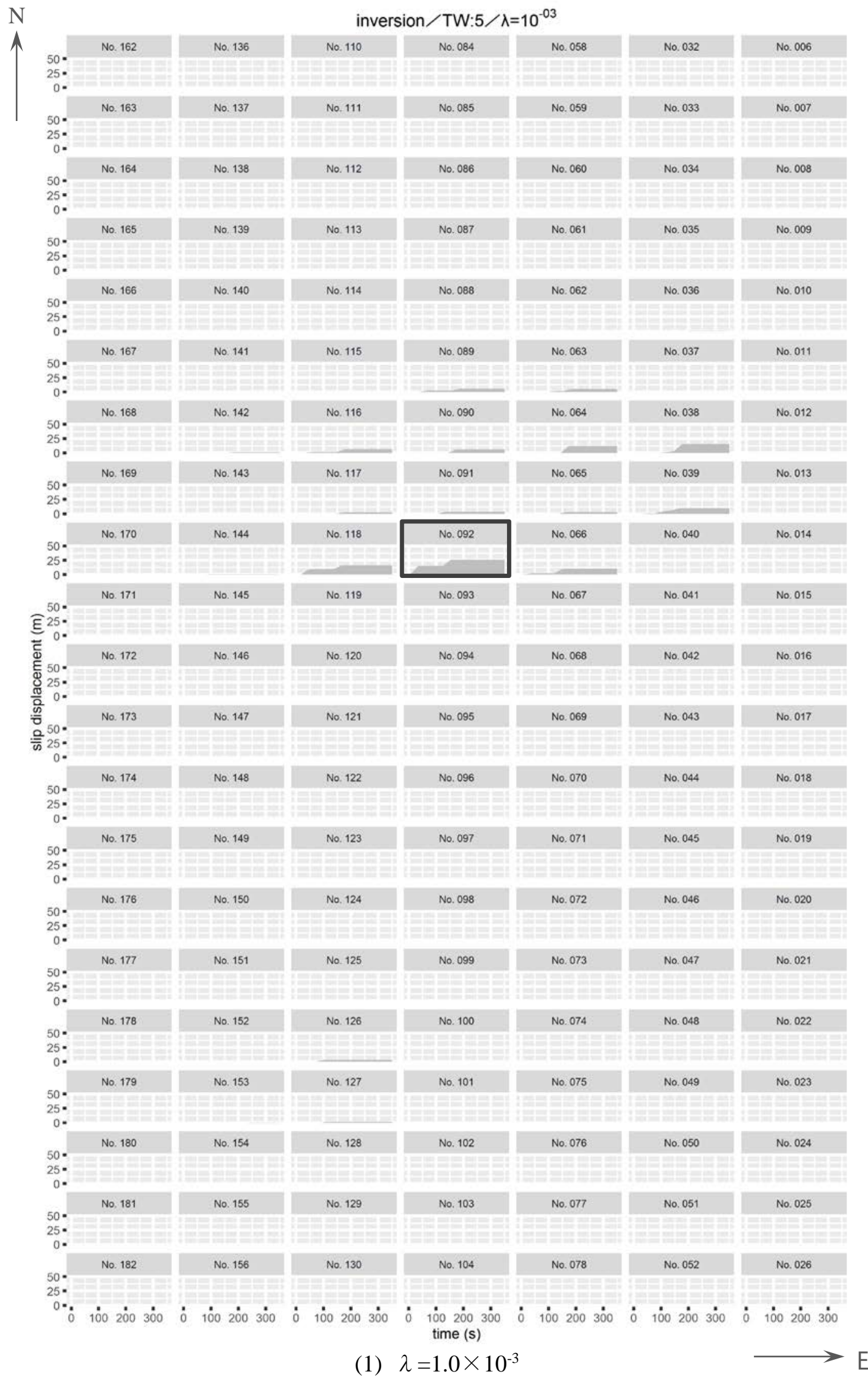

Fig. 6 Lasso regression estimate of slip block time change of fault block (1) 


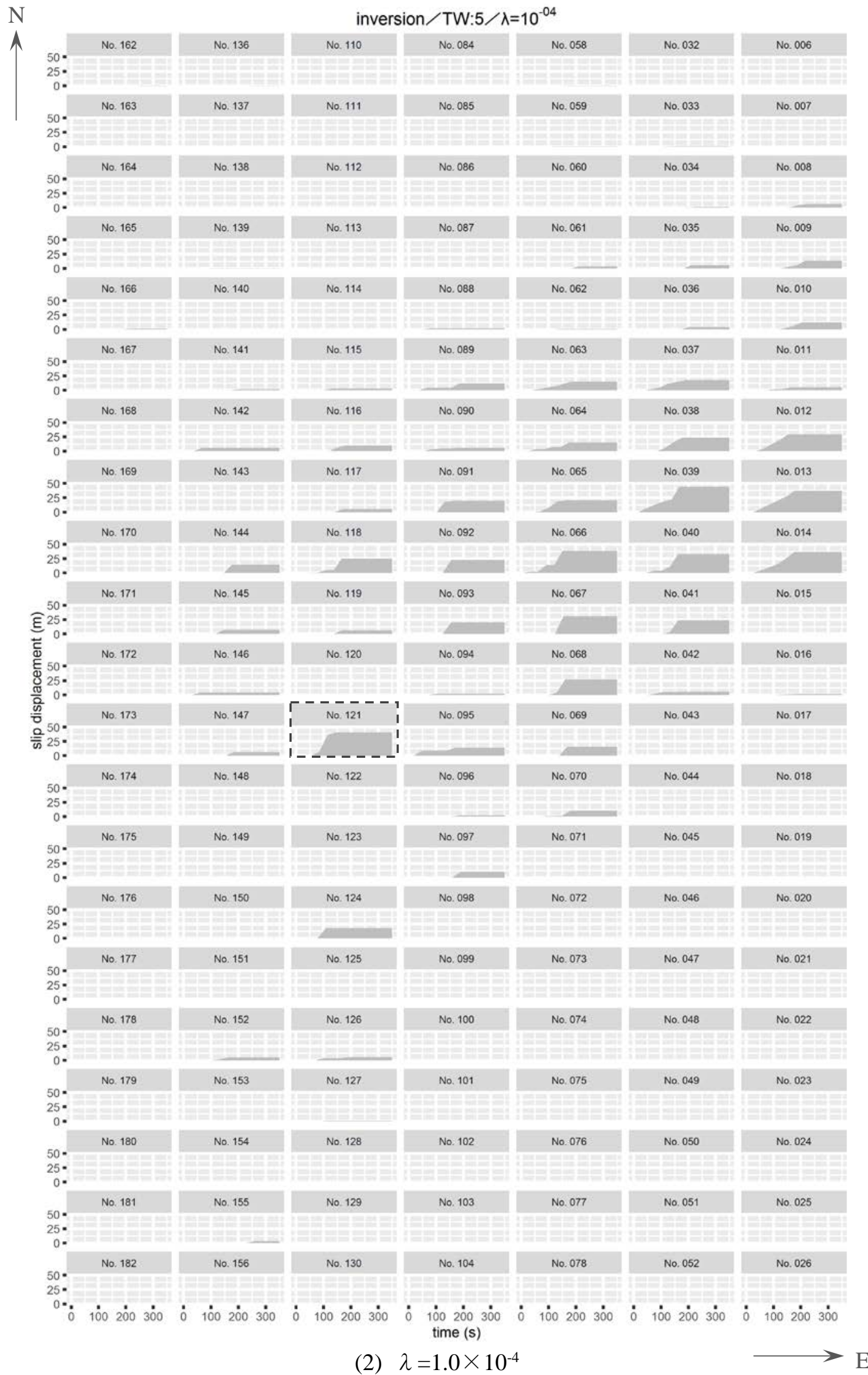

Fig. 6 Lasso regression estimate of slip block time change of fault block (2) 


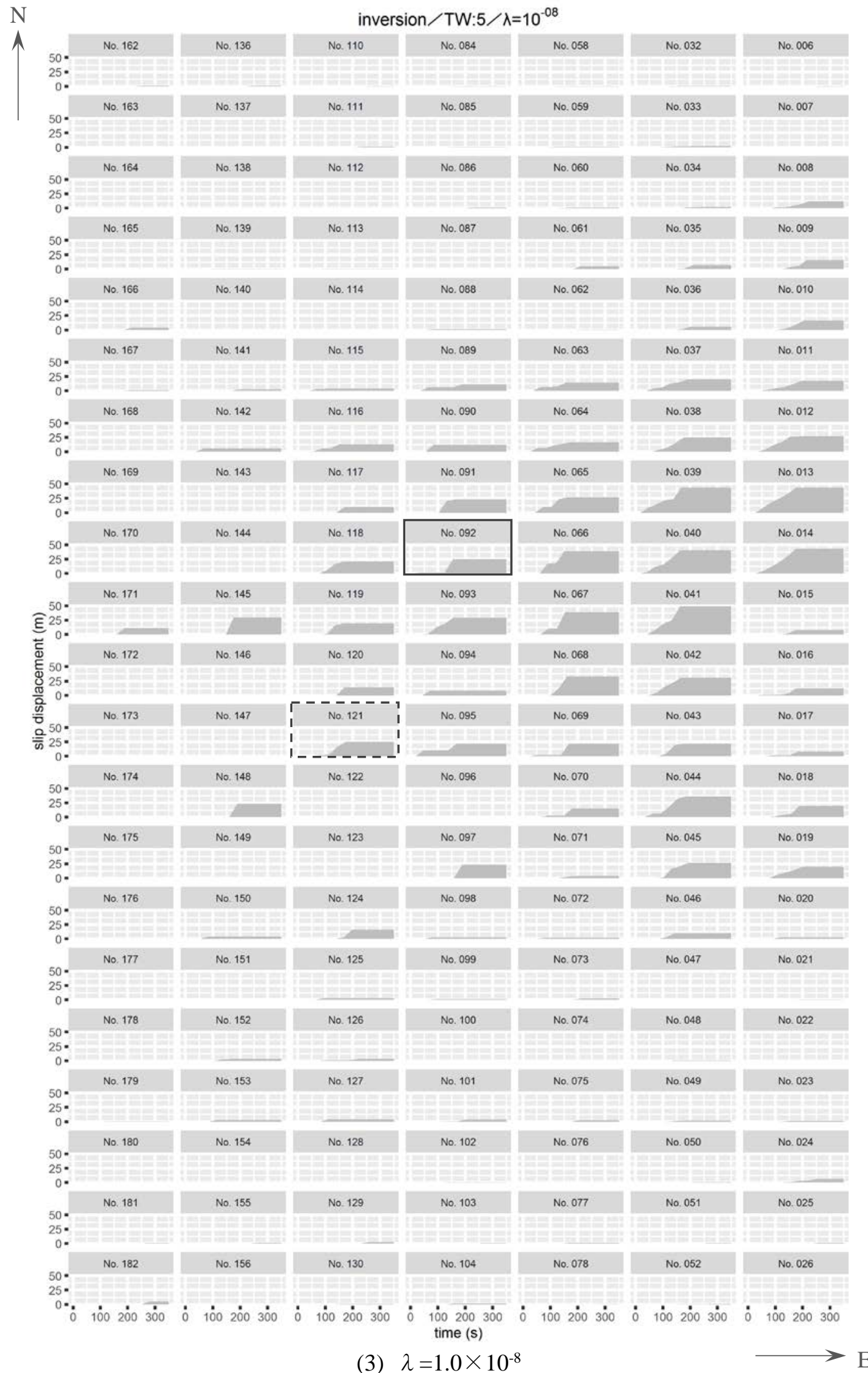

Fig. 6 Lasso regression estimate of slip block time change of fault block (3) 
solution path of slip displacement/TW:No.1

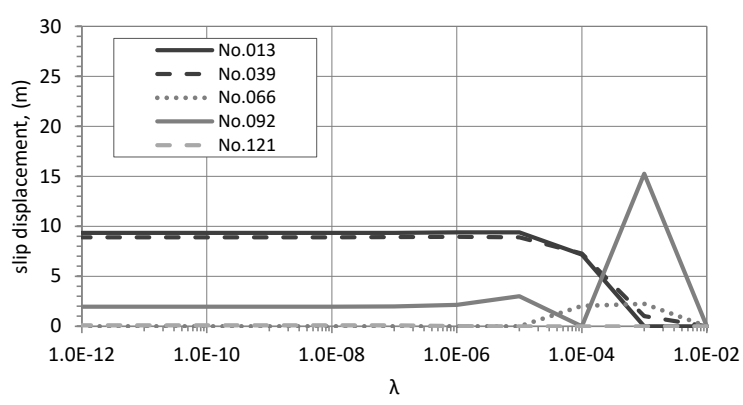

(1) Time window (TW) : 1

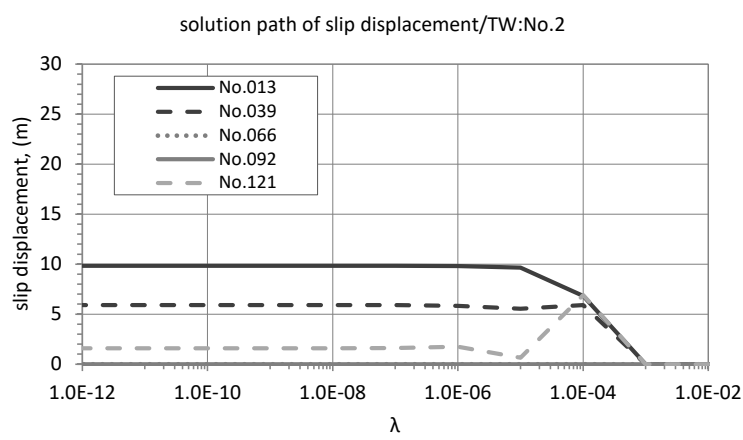

(2) Time window (TW) : 2

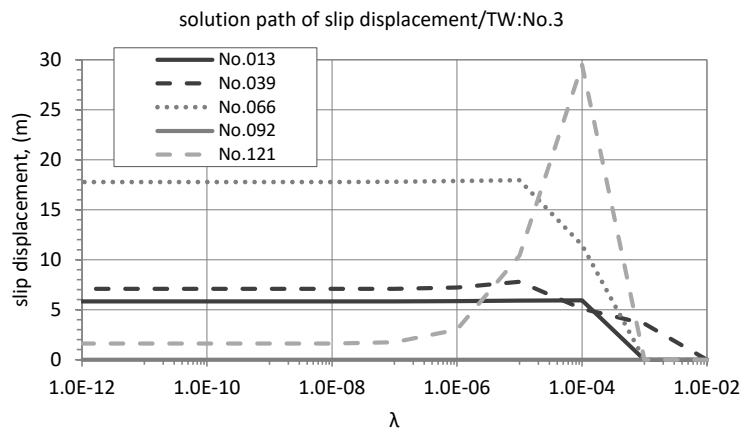

(3) Time window (TW) : 3

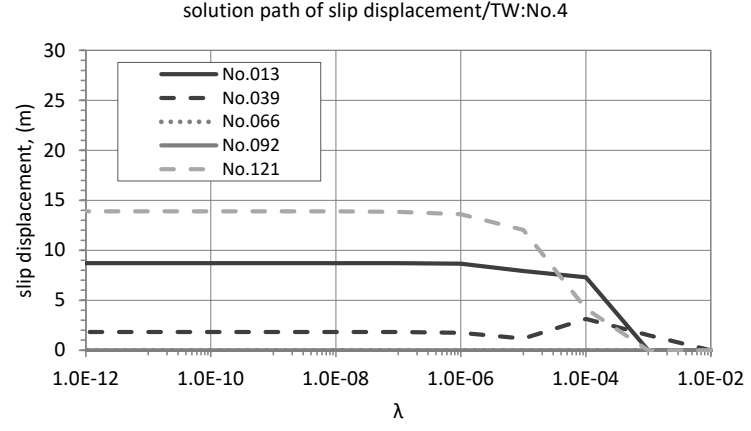

(4) Time window (TW) : 4

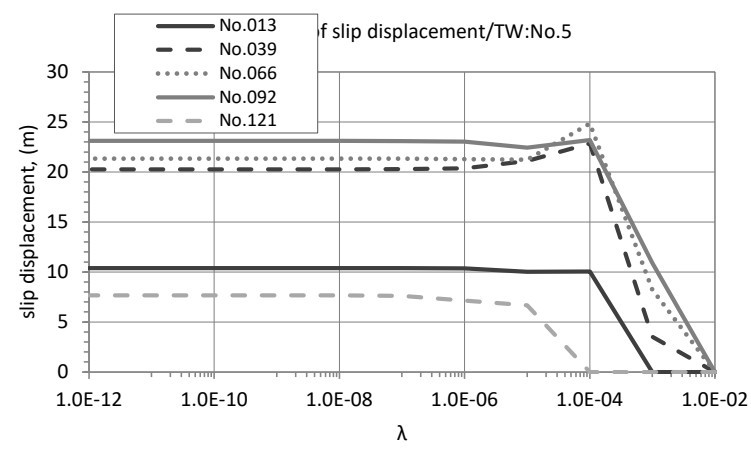

(5) Time window (TW) : 5

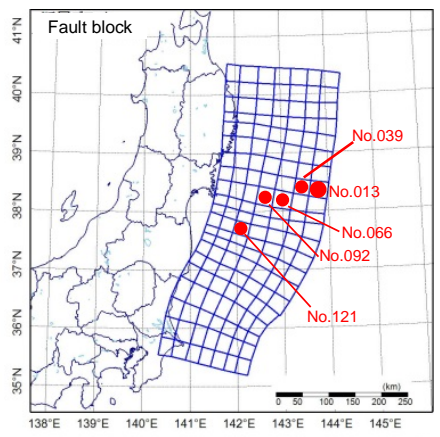

(6) Fault block for solution path

Fig. 7 Solution path of fault slip by Lasso regression

\section{DISCUSSION}

Using the source fault model obtained using sparse modeling, the reproducibility of the time history waveform of the tsunami water level was analyzed. The following discussion of the important factors in constructing a prediction model for earthquake faults will focus on this reproducibility. In addition, although the crustal movement (permanent displacement) and the tsunami water level time history are used as observation data in the tsunami inversion of this study, focus is placed on the time history of tsunami water level since the tsunami water level is more important in the prediction problem in tsunami-resistant design. The observation points of the tsunami water level time history used here are shown in Fig. 8. 


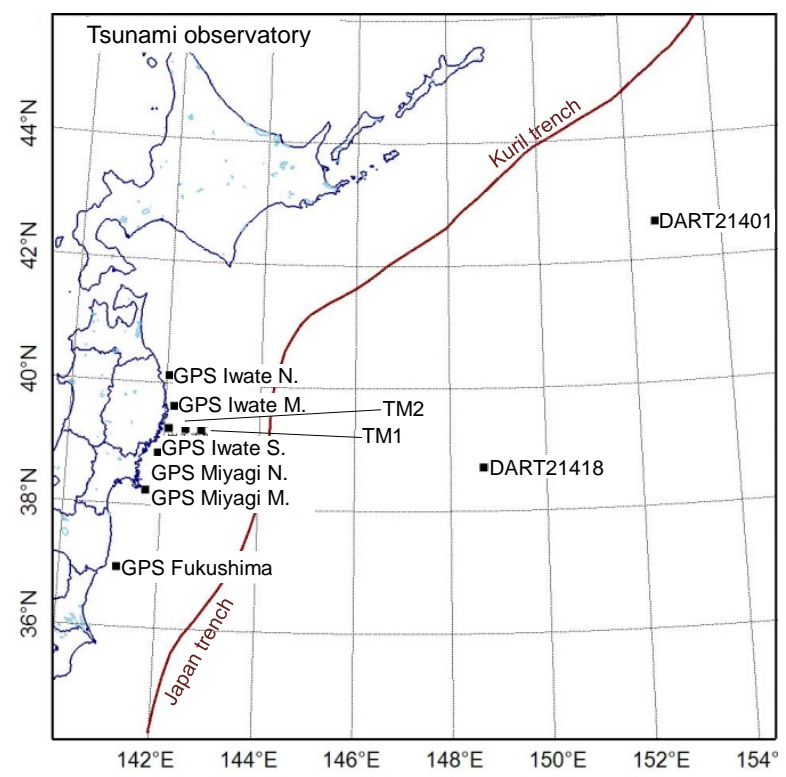

Fig. 8 Stations used to investigate the tsunami water level time history

Figure 9 shows the time history of tsunami simulation results using the estimated fault slip model obtained by performing a Lasso regression by changing the value of the regularization parameter. Here, the simulation of tsunami water level time history was carried out by solving the nonlinear long wave equation discretized by the finite difference method (FDM). Figure 9 shows that the amplitude of the tsunami water level time history using the Lasso regression results is clearly smaller than that of the observation record in the case of $\lambda=1.0 \times 10^{-3}$. On the other hand, in the case of $\lambda=1.0 \times 10^{-8}$, the tsunami water level time history by the inversion result and the observation record are quite consistent. The examination of the slip characteristics of the fault model will proceed based on the quantitative relationship between the regularization parameter values and the tsunami water level time history.

In the case of $\lambda=1.0 \times 10^{-3}$ in Fig. 9 (1), the arrival time of the first direct wave coincides with observed data. On the other hand, the characteristic sharp pulse waveform in the time history cannot be expressed at all. However, the relatively long period components which constitute the basis of the waveform are consistent with the observation records. Therefore, in the fault model shown in Fig. 3 (2) and Fig. 6 (1), the area where slips are concentrated in a near square shape contributes largely to the arrival time of the first wave and the long period components that constitute the base of the waveform. Next, as shown in Fig. 3 (3) and Fig. 6 (2), in the case of fault model $\left(\lambda=1.0 \times 10^{-4}\right)$, in which the slip has developed to the near trench axis, a sharp pulse waveform in the time history is expressed like the time history in Fig. 9 (2). In the individual detailed comparison of time history, although there are still variances from the recorded observations, the compatibility of both is improved significantly. Finally, the increase in the slip distribution area in space and time, as shown in Fig. 3 (4) to (8) and Fig. 6 (3), leads to a fault model that is highlight consistent with observation records, as shown in Fig. 9 (3) to (4).

Figure 10 shows the amplitude ratio of time histories of tsunami simulation results using the Lasso regression model and observed values. Here, two types of calculation methods are shown: the maximum amplitude ratio and the RMS amplitude ratio. The results of both demonstrate virtually the same tendency. The amplitude ratio at $\lambda=1.0 \times 10^{-3}$ is about 0.4 . When $\lambda=1.0 \times 10^{-6}$ or less, the fluctuation of the amplitude ratio does not occur and converges to a steady value. Also, regardless of the value of $\lambda$, the value of the amplitude ratio varies from observation point to observation point. As shown in Fig. 3 (2), fault slips at $\lambda=1.0 \times 10^{-3}$ exist in the limited region. Nevertheless, the fact that about $40 \%$ of the time history waveform can be explained is considered to be that the amount of slip in this region has a considerable influence on the overall behavior. 


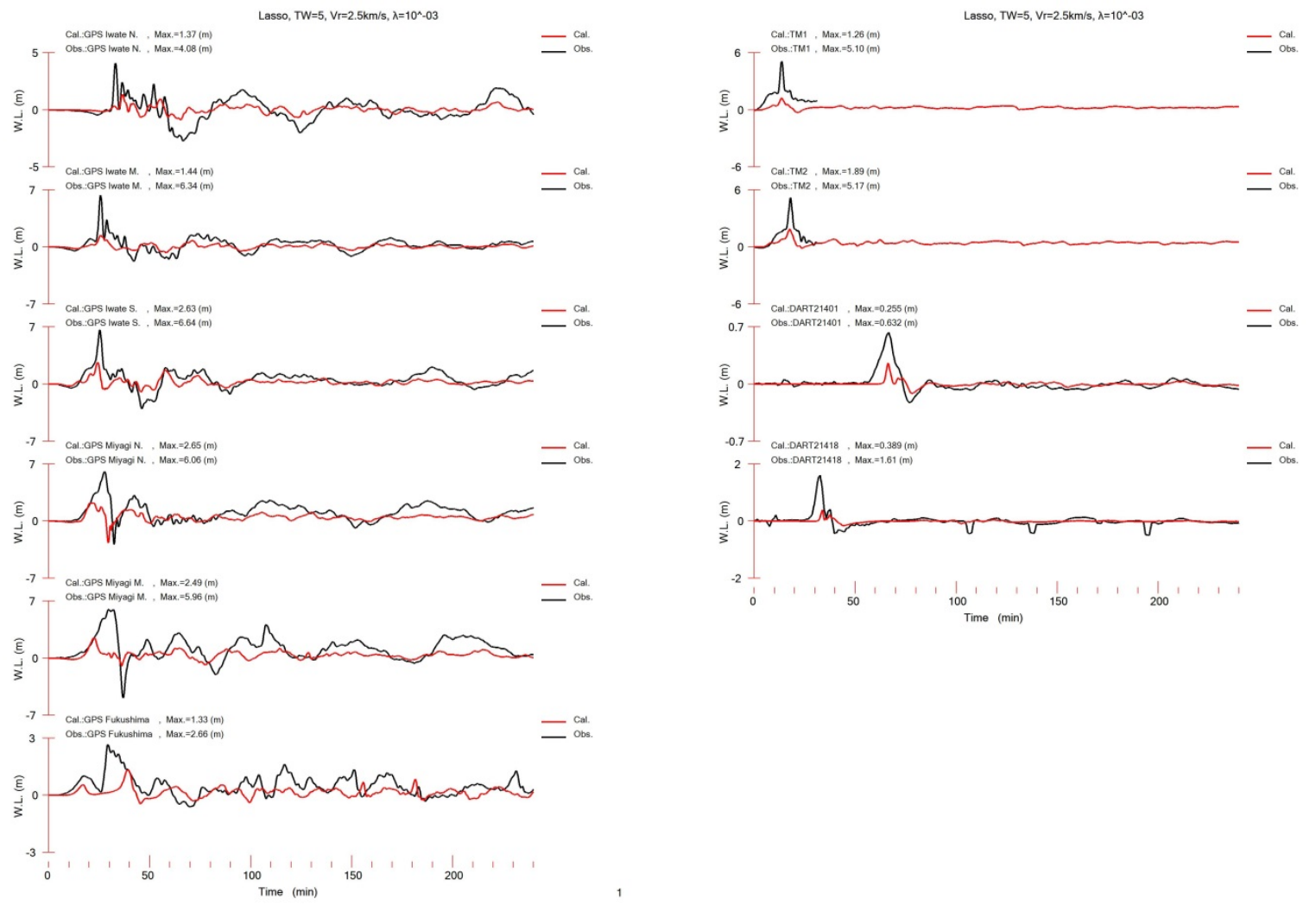

(1) $\lambda=1.0 \times 10^{-3}$

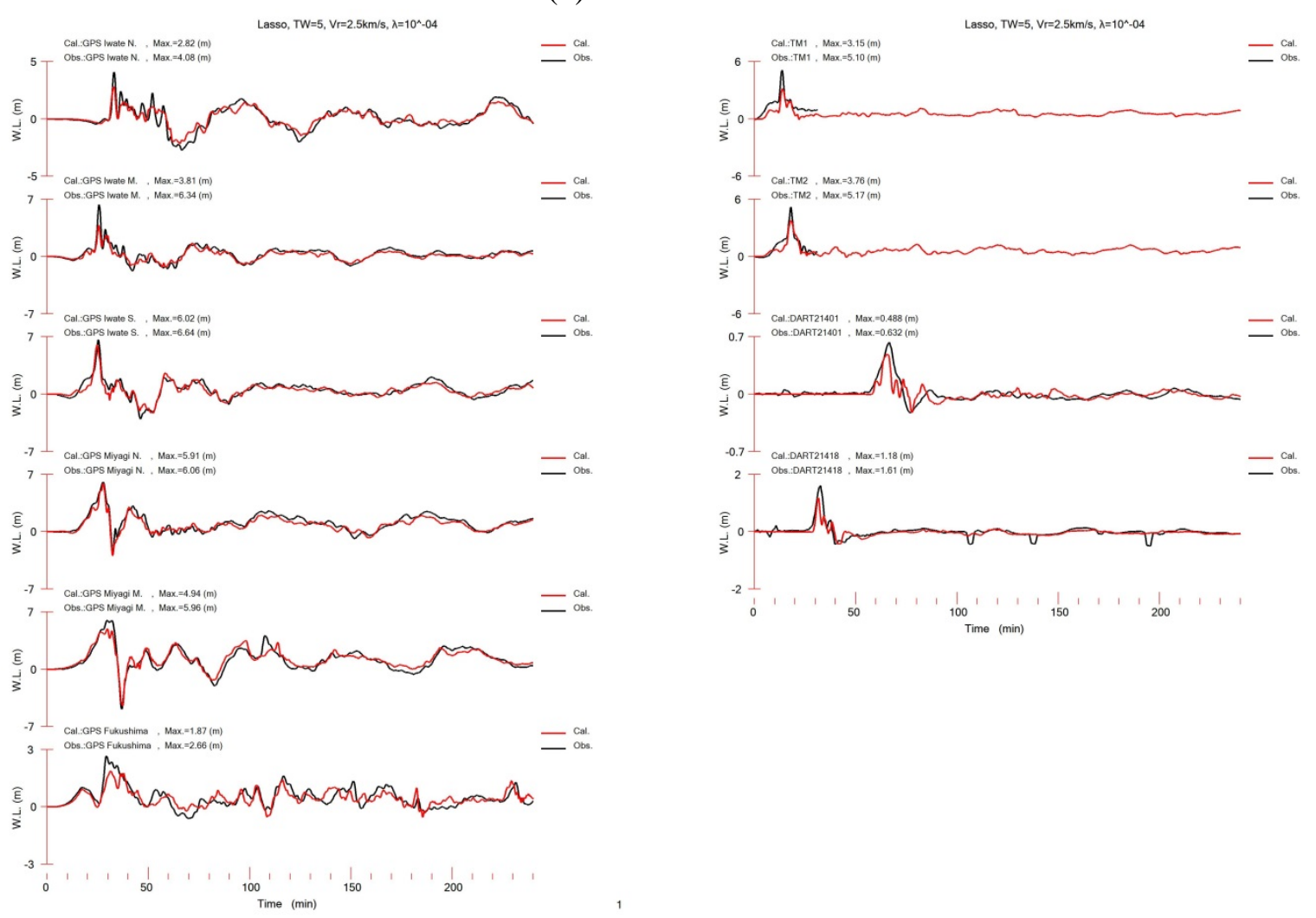

(2) $\lambda=1.0 \times 10^{-4}$

Fig. 9 Time history of tsunami simulation results using the fault slip model estimated using a Lasso regression (1) 


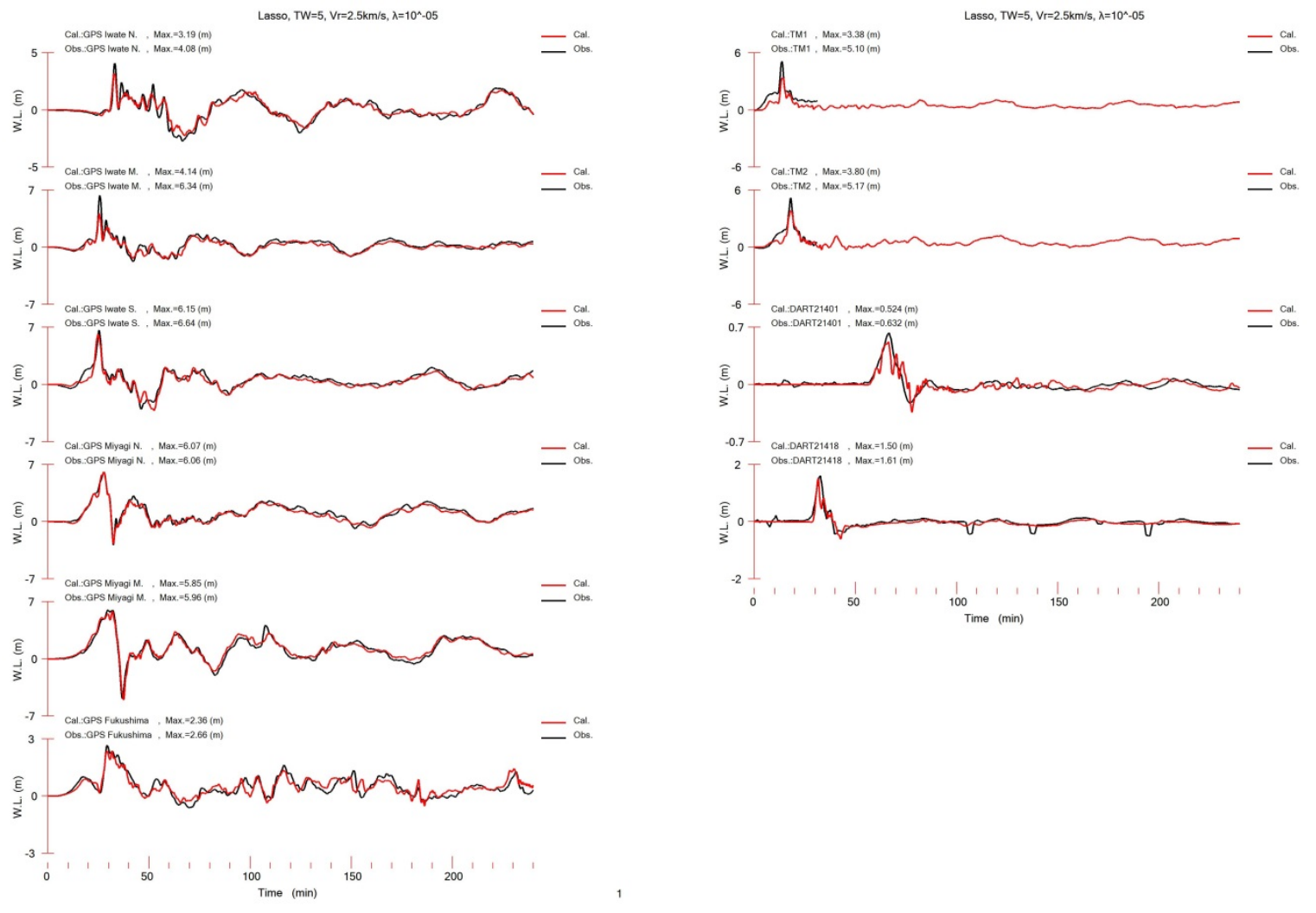

(3) $\lambda=1.0 \times 10^{-5}$

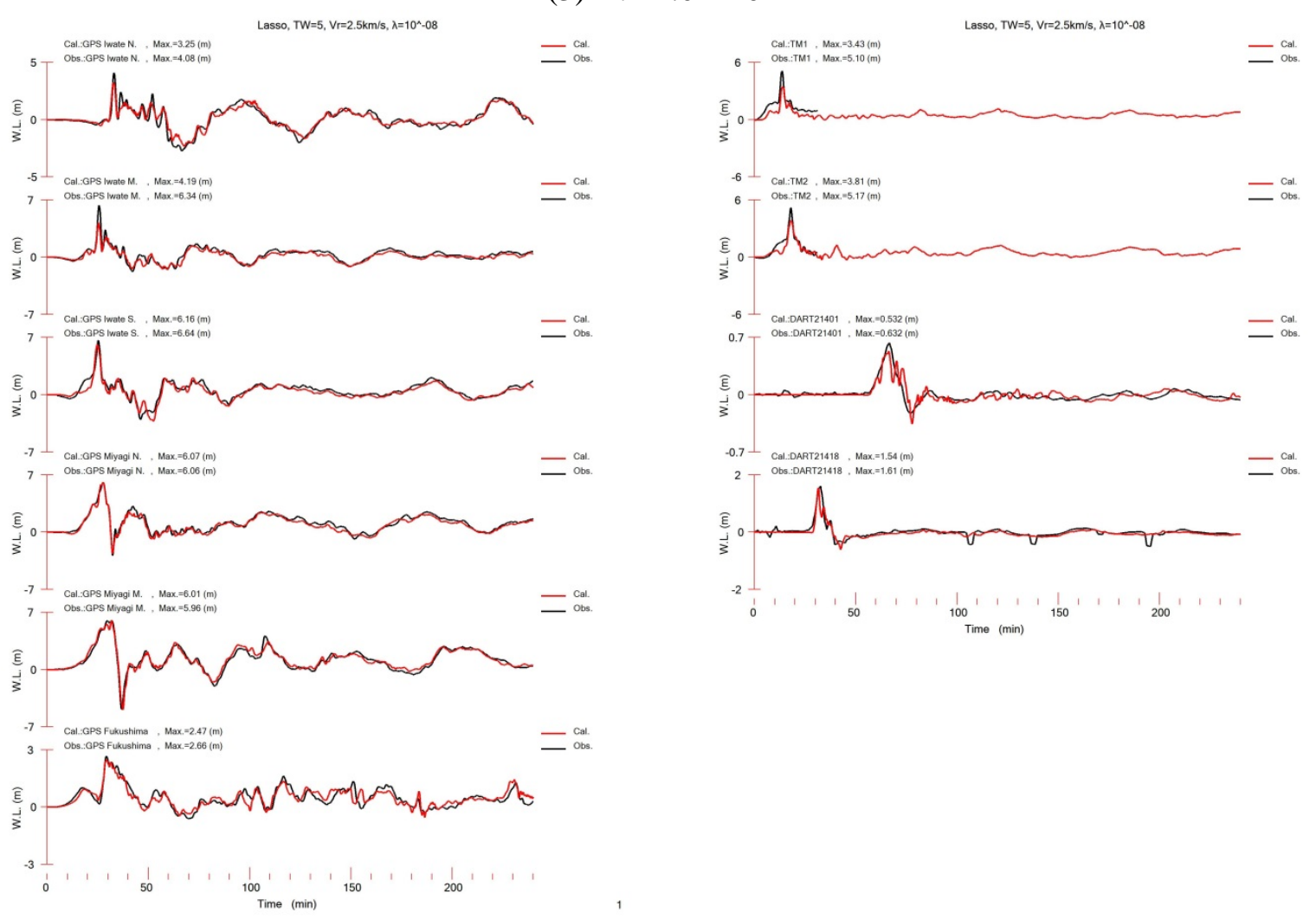

(4) $\lambda=1.0 \times 10^{-8}$

Fig. 9 Time history of tsunami simulation results using a fault slip model estimated using a Lasso regression (2) 


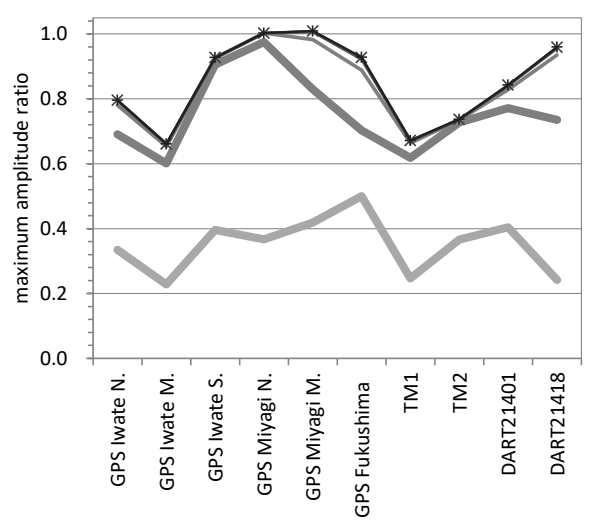

(1) Maximum amplitude ratio

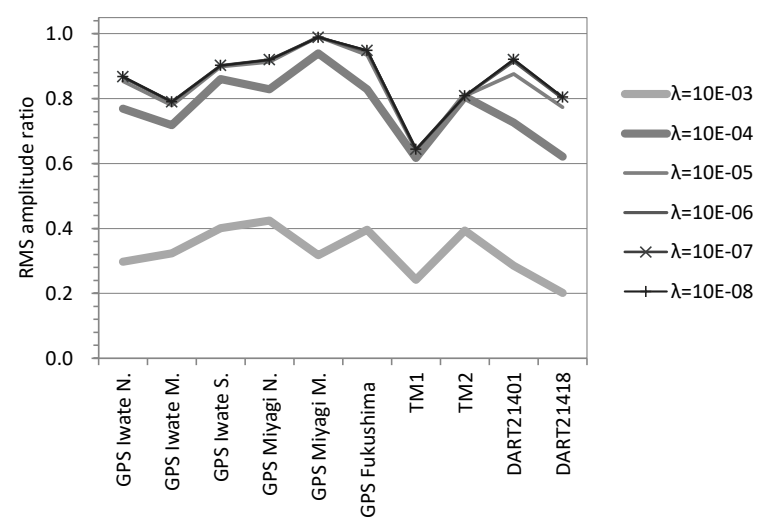

(2) RMS amplitude ratio

Fig. 10 Amplitude ratio of time history of tsunami simulation result using a Lasso regression model and observed value

transitional rss of time history

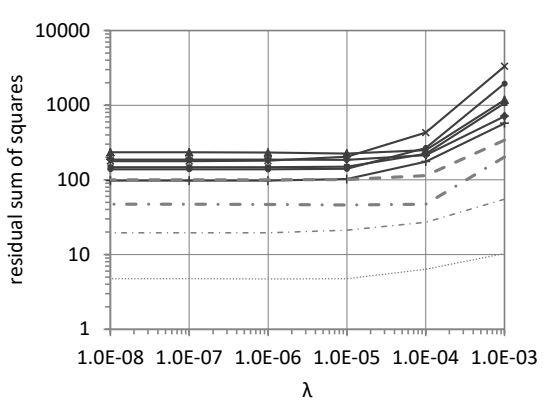

(1) Residual sum of squares

transitional correlation coeff. of time history

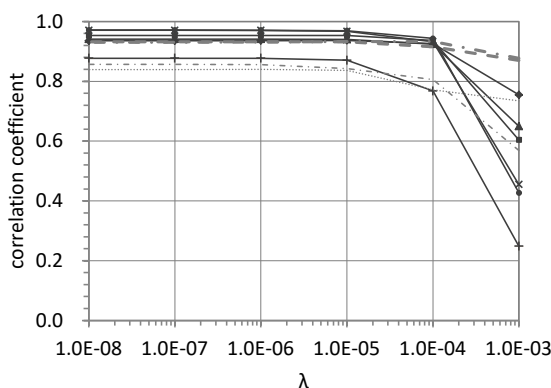

(3) Correlation coefficient

transitional M of time history

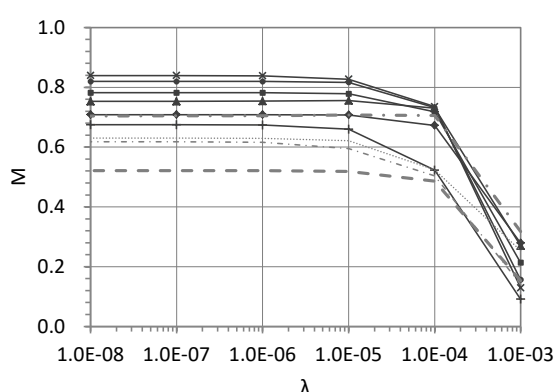

transitional nRMSE of time history

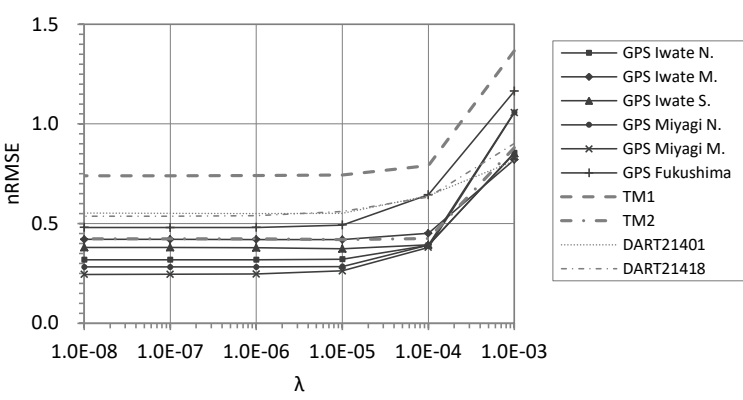

(2) normalized RMSE (nRMSE)

transitional IOA of time history
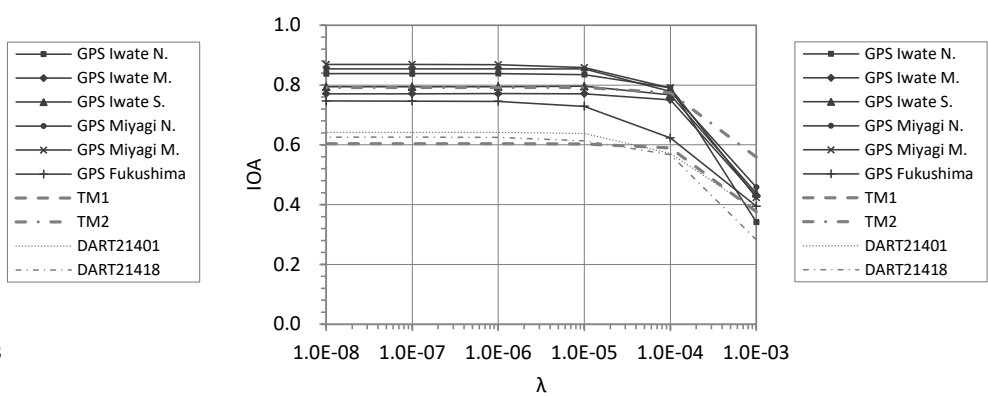

(4) Compatibility index by Willmott et al. (1985) (IOA)

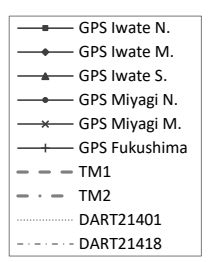

transitional R of time history

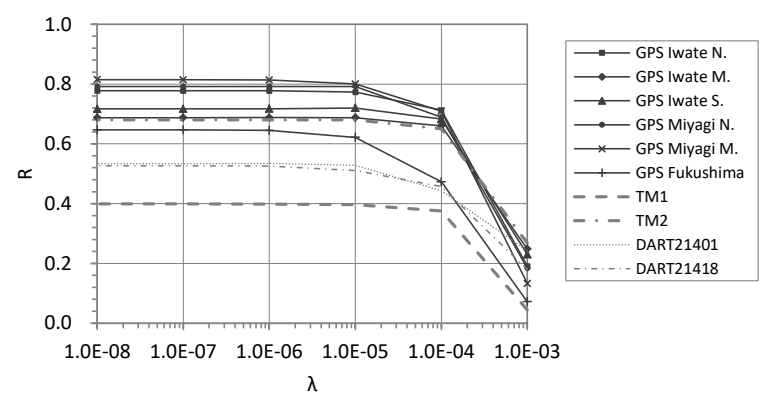

(5) Index of Watterson's (1996) transformed Mielke (M) (6) Index of Mielke and Berry (2001) (R)

Fig. 11 A compatibility index of time history between tsunami simulation results using a Lasso regression model and observed values 
The following explanations may be derived from the results presented thus far. A relatively long period component of the tsunami water level time history is generated by fault slips at a fairly deep part of the plate boundary off Miyagi Prefecture, which contributes to the formation of the main part of the waveform. Since relatively short period waves are generated from the tsunami earthquake area near the trench axis, sharp pulses and small undulations of the tsunami water level time history may be caused by the slip in this area. The application of the proposed method produced results that support the results of previous studies ${ }^{\text {e. g. } 6 \text { ). }}$

Figure 11 shows the results obtained using various indices for the time history compatibility of the observed values and tsunami simulation results using a Lasso regression model with each regularization parameter. The indices used in Allen and Greenslade ${ }^{18)}$ were followed as indices to evaluate the compatibility of observation records and numerical simulation results for tsunami water level time history. Allen and Greenslade ${ }^{18)}$ found that the indices such as IOA and $\mathrm{M}$ are more suitable for evaluating the compatibility of time history waveforms, since they are more sensitive to waveform differences compared to correlation coefficients and nRMSE. In that same paper, they were used for the comparing the results of numerical calculations, but in this study, they are being used to compare observed values and numerical simulation results.

Figure 11 shows that with the residual sum of squares and nRMSE, the difference between the wave reproduced by the numerical simulation and the observed data increases as the regularization parameter increases, but it is difficult to present versatile standard values for the compatibility of either. Since the maximum value of the other indicators is 1.0, they are deemed appropriate for a quantitative determination of compatibility. For these indices, the results show that the compatibility of either index is poor when the regularization parameter is at its largest $\left(\lambda=1.0 \times 10^{-3}\right)$. However, as the regularization parameter gradually decreases and the waveform difference decreases, the sensitivity of the other three indices (IOA, $\mathrm{M}, \mathfrak{R}$ ) are shown to be more sensitive to the difference in the model than the correlation coefficient, which is approximately 0.8 or more. Furthermore, the time history waveforms of the simulation results of TM1 and TM2 at $\lambda=1.0 \times 10^{-3}$ in Fig. 9 (1) and the observed values do not seem to correspond well. However, the correlation coefficient is a sufficiently large value of about 0.87 . This indicates that visual sense and correlation coefficient values do not always match. At this time, the three indexes of IOA, $M$ and $\mathfrak{R}$ are less than 0.5 at most of the observation points, which indicates that the compatibility is not high. The compatibility for these three indices is higher when the regularization parameter is smaller than $\lambda=1.0 \times 10^{-4}$. This result is consistent with the visual impression received from the compatibility of the time history waveforms in Figs. 9 (2) to (4).

According to the three indices of IOA, $\mathrm{M}$ and $\mathfrak{R}$, the order of the quality of the compatibility is consistent when the regularization parameter is small. GPS Miyagi M. has the highest compatibility, followed by GPS Miyagi N. and GPS Iwate N. Of the GPS ocean wave meter, the least compatible is GPS Fukushima. Among all the observation points, TM1 is the least compatible, followed by DART 21418 and DART 21401. TM1 is caused by the fact that the reproducibility of the amplitude of the pulse part that produces the maximum value is not sufficient. The DART seems to be influenced by the inclusion in the simulation result of a short period component not found in the observation data.

In addition, when evaluating the compatibility between the simulation results using the fault model obtained by tsunami inversion and the observation records, indices like IOA, $M$ and $\mathfrak{R}$, introduced in Allen and Greenslade ${ }^{18)}$ are more useful than the correlation coefficient when examining models because they reflect differences in tsunami waveforms derived from different fault models.

When performing future tsunami predictions necessary for tsunami-resistant design, the characterized fault model ${ }^{19}$ ) is generally used as the model of the wave source. Constructing a characterization fault model requires that fault plane be divided into a super-large slip area, large slip area, and background area, based on the slip displacements. For such segmentation, it is necessary to carry out inversion analysis for past tsunamis and to examine the characteristics of the slip distribution in the earthquake source fault. It is important to accumulate such information. 
By performing tsunami inversion based on the concept of sparse modeling, as described above, it is possible to efficiently understand which part of the spatiotemporal slip model of the seismic source fault contributes to the tsunami waveform. In particular, the results showed that the main characteristics of the tsunami waveform are formed by the slip that occurs at a slightly deeper portion of the plate boundary, and the slip near the trench axis contributes to the short period components of the tsunami waveform. The proposed method was therefore shown to be effective in analyzing the slip characteristics of fault models.

\section{CONCLUSIONS}

In this study, tsunami inversion was carried out using a Lasso regression, which is a typical form of sparse modeling, and the characteristics of the spatiotemporal distribution of slips in the seismic source fault were analyzed. This was done for the key purpose of developing the kind of tsunami prediction model necessary for tsunami-resistant design. The conclusions of this study derived from the investigation results are summarized below.

- Lasso-type sparse modeling can be applied to the tsunami inversion problem by modifying the conventional soft-thresholding function and using the soft-thresholding function that satisfies the nonnegative condition.

- By increasing the value of the regularization parameter, the solution of fault slip that has a large impact on the observation values, such as tsunami waveform, was obtained. This is considered to be important information in analyzing where and at which time the result of the slip has the most significance in the tsunami phenomenon on the fault plane.

- The results of the tsunami inversion based on the concept of sparse modeling show that the main characteristic of the tsunami waveform is formed by the slip occurring at a slightly deeper part of the plate boundary, and that slip near the trench axis contributes to relatively short-period components such as sharp pulses and small undulations in the tsunami waveform.

- An evaluation of the compatibility between the observation records and the simulation results using the fault models obtained by tsunami inversion, revealed that the indices (e.g., IOA, M and $\Re$ ) introduced in Allen and Greenslade ${ }^{18)}$ are more useful than the conventionally used correlation coefficients or RMSE when examining the models, because they more sensitively reflect differences in tsunami waveforms in different fault models.

\section{REFERENCES}

1) Kurita, T., Matsuyama M. and Uchino D.: Uncertainty of Numerical Tsunami Simulation Evaluated in Comparison with the Field Survey results of the 2011 Tohoku Earthquake Tsunami, Journal of JSCE, Ser. B2 (Coastal Engineering), Vol. 69, No. 2, pp. I_216-I_220, 2013. (in Japanese)

2) Tomioka, R.: Machine Learning with Sparsity Inducing Regularizations, Machine Learning Professional Series, Kodansha Ltd., 2015. (in Japanese)

3) Hontani, H.: Image processing and sparse, Sparse modeling and multivariate data analysis, Iwanami Data Science, Vol. 5, pp. 64-79, Iwanami Shoten, Publishers, 2017. (in Japanese)

4) Iba, Y.: Model selection hyper-practice, Sparse modeling and multivariate data analysis, Iwanami Data Science, Vol. 5, pp. 6-18, Iwanami Shoten, Publishers, 2017. (in Japanese)

5) Kurita, T.: Inversion of Crustal Deformation and Tsunami Waveform Data for Fault Slip Models, Journal of JSCE, Ser. A2 (Applied Mechanics), Vol. 72, No. 2, pp. I_13-I_23, 2016. (in Japanese)

6) Satake, K., Fujii, Y., Harada, T. and Namegaya Y.: Time and Space Distribution of Coseismic Slip of the 2011 Tohoku Earthquake as Inferred from Tsunami Waveform Data, Bulletin of the Seismological Society of America, Vol. 103, No. 2B, pp. 1473-1492, 2013.

7) Tibshirani, R.: Regression Shrinkage and Selection via the Lasso, Journal of Royal Statistics 
Society, Series B, Vol. 58, pp. 267-288, 1996.

8) Friedman, J., Hastie, T., Hofling, H. and Tibshirani, R.: Pathwise Coordinate Optimization, The Annals of Applied Statistics, Vol. 1, No. 2, pp. 302-332, 2007.

9) Friedman, J., Hstie, T. and Tibshirani, R.: Regularization Paths for Generalized Linear Models via Coordinate Descent, Journal of Statistical Software, Vol. 33, No. 1, 2010.

10) Boyd, S., Parikh, N., Chu, E., Peleato, B. and Eckstein, J.: Distributed Optimization and Statistical Learning via the Alternating Direction Method of Multipliers, Foundations and Trends ${ }^{\circledR}$ in Machine Learning, Vol. 3, No. 1, pp. 1-122, 2011.

11) Geospatial Information Authority of Japan: Crustal deformation associated with the March 11, 2011 main shock (Mw9.0), Crustal deformation of electronic reference point obtained from GPS continuous observation, http://www.gsi.go.jp/BOUSAI/h23_tohoku.html, 2011. (in Japanese, last accessed on July 24, 2012)

12) Japan Coast Guard: Seafloor movement associated with the 2011 Tohoku earthquake - Seafloor crustal movement observation results -, http://www1.kaiho.mlit.go.jp/jishin/11tohoku/index.html, 2011. (last accessed on July 24, 2012)

13) Japan Meteorological Agency: Real time tide data, http://www1.kaiho.mlit.go.jp/KANKYO/TIDE/real_time_tide/sel/index.htm, 2011. (in Japanese, last accessed on April 13, 2011)

14) Ports and Harbours Bureau, Ministry of Land, Infrastructure, Transport and Tourism: NOWPHAS (Nationwide Ocean Wave information network for Ports and Harbours), http://www.mlit.go.jp/kowan/nowphas/index.html, 2011. (last accessed on April 13, 2011)

15) National Oceanic and Atmospheric Administration: National Data Buoy Center, http://nctr.pmel.noaa.gov/Dart/, 2011. (in Japanese, last accessed on April 11, 2011)

16) Earthquake Research Institute, University of Tokyo: Sea-level change recorded by the ocean floor cable seismometer off Kamaishi, http://www.eri.u-tokyo.ac.jp/PREV_HP/outreach/eqvolc/201103_tohoku/\#kamaishimeter, 2011. (last accessed on April 15, 2011)

17) Ninomiya, Y. and Kawano, S.: AIC for the Lasso in Generalized Linear Models, Electric Journal of Statistics, Vol. 10, pp. 2537-2560, 2016.

18) Allen, S. C. R. and Greenslade, D. J. M.: Indices for the Objective Assessment of Tsunami Forecast Model, Pure and Applied Geophysics, Vol. 170, Nos. 9-10, pp. 1601-1620, 2013.

19) Tsunami Evaluation Subcommittee, Nuclear Civil Engineering Committee, Japan Society of Civil Engineers: Tsunami Assessment Method for Nuclear Power Plants in Japan 2016, 2.2.5 Examples of basic fault model setting method, pp. Appendix 2-63 - Appendix 2-68, 2016. (in Japanese)

(Original Japanese Paper Published: May, 2019) (English Version Submitted: July 2, 2019) (English Version Accepted: July 25, 2019) 